\section{New malaria vaccine shows promise}

Deborah Josefson, San Francisco

An experimental malaria vaccine designed to protect the host from each stage of the parasite's life cycle has shown promise in animal trials (Proceedings of the National Academy of Sciences 1999;96:1615-20).

Researchers from the US Centers for Disease Control and Prevention in Atlanta, Georgia, have created a recombinant, multivalent vaccine against Plasmodium falciparum, the deadliest malaria parasite. This vaccine differs from previous vaccines because it contains multiple antigens and targets the parasite at several stages in its life cycle.

Malaria infects about 400 million people a year and kills 3 million annually. The development of an effective malaria vac- cine has become an international health priority.

Construction of a successful vaccine has been hampered by the complexity of the parasite's life cycle. In addition to both the mosquito vector and vertebrate phases, the parasite undergoes multiple incarnations within its host. For instance, it has an asexual sporozoite phase in which it freely circulates within the bloodstream, a gametocytic phase within red blood cells, a schizont phase, and a merozoite phase within hepatocytes. The parasite's identity changes through each of these stages-including changes in surface antigens-and this presents a challenge to vaccine development.

Additionally, the parasite cloaks itself in different proteins during the erythrocytic and hepatocytic phases, and these membrane proteins also undergo phenotypic variation. Therefore a vaccine directed against a single epitope or which targets only one phase of the parasite's life cycle may not be sufficient to induce protective antibodies.

The centres' scientists, led by Altaf Lal, took advantage of the parasite's complicated life stages by constructing a vaccine which combined segments of 21 different $P$ faliciparum peptides into a single recombinant protein. The peptides were selected because each of them had been shown to be immunogenic in previous vaccine trials.

Different parts of the host's immune system-B cells, helper $\mathrm{T}$ cells, and cytotoxic $\mathrm{T}$ cells-were activated by the peptides.

The scientists plan to test the vaccine on non-human primates before proceeding to human trials.

\section{Staphylococcus resistant to vancomycin emerges}

\section{Scott Gottlieb New York}

Researchers have reported the first three cases in the United States of infections caused by Staphylococcus aureus bacteria resistant to vancomycin, an antibiotic often reserved to fight these infections when no other agent is effective.

To date, a total of four strains of $S$ aureus intermediately resistant to vancomycin have been documented worldwide, the first in Japan in 1996.

Certain common factors in the US cases suggest that cellular modification as a result of prolonged exposure to vancomycin was probably responsible for the emergence of the recent resistant isolates.

These strains "threaten to return us to the era before the development of antibiotics," warn researchers at the US Centers for Disease Control and Prevention in Atlanta, Georgia (New England Journal of Medicine 1999;340:493-501;517-23;556-7).

$S$ aureus, a common bacterium, is easily transmitted through

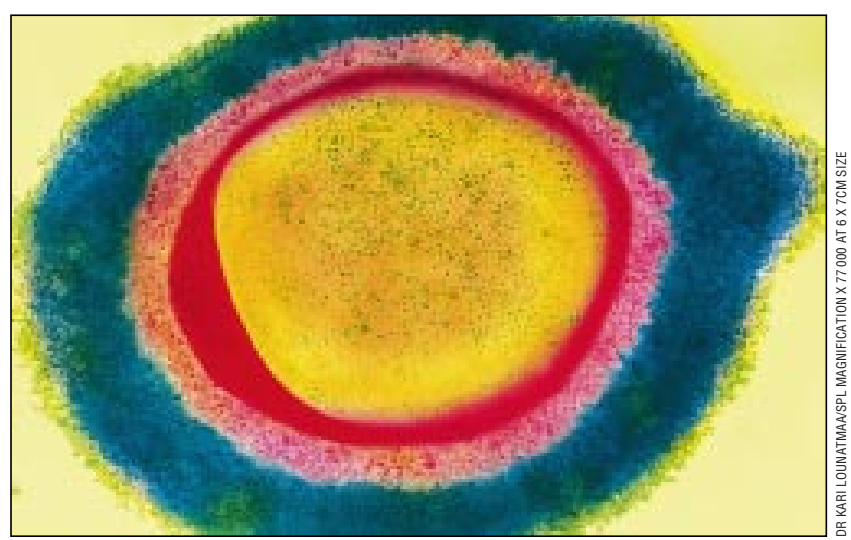

S aureus-vancomycin won't always stop it

physical contact with infected people. It is a common cause of skin infections, such as infections of surgical wounds, and also of sepsis.

It has become resistant to a wide range of antibiotics, however, and vancomycin was considered an important "last resort." The patients whose clinical courses were reported in two separate articles in the New England Journal of Medicine each had underlying conditions, including infections with methicillin resistant strains of $S$ aureus that were given vancomycin intravenously for extended periods.

All three infections were eventually brought under control by using combinations of various other antibiotics, but in each case, the patients died anyway. Penicillin type antibiotics have proved effective against infection with $S$ aureus in the past.

The general overuse of antibiotics has, however, allowed the infection to develop mutations, rendering it resistant to many of these drugs.

The current studies suggest that the bacterium has developed an alternative vancomycin binding pathway that diverts vancomycin away from the usual target site.

The researchers point out that all three US patients had histories of long term treatment with vancomycin linked with dialysis.

\section{Scottish white paper aims to tackle health divide}

Bryan Christie, Edinburgh

A sustained attack on inequality, social exclusion, and poverty is promised in a government white paper on public health in Scotland which identifies these factors as the root causes of much of the country's ill health.

Although there have been improvements in recent years, Scotland still has some of the highest rates of disease in the developed world. The report sets out a strategy for tackling the problem, which is based as much on investment in housing, education, and employment opportunities as it is on health related services.

Sam Galbraith, the Scottish health minister, said that he hoped the programme would have the same positive impact on health in the 21st century as improving public sanitation and slum housing did at the beginning of the 20th century.

Particular initiatives are directed at improving the detection and prevention of disease. A total of $£ 15 \mathrm{~m}$ is to be spent on four "demonstration" projects which will aim to reduce premature deaths from cancer, cut deaths from heart disease, improve child health, and reduce teenage pregnancies.

New targets have been set to reduce the number of adults dying from heart disease by $50 \%$ by the year 2010 and cancer deaths among those aged under 75 by $20 \%$.

A pilot project will examine the feasibility of introducing a national screening programme for colorectal cancer, and smokers are to be helped to give up the habit at specialist clinics.

An additional $£ 34.5 \mathrm{~m}$ is to be spent on establishing a network of healthy living centres, and a task force is to be set up to develop a national physical activity strategy. Funding is also to be increased for initiatives to improve diet, and support is given in the white paper for the fluoridation of drinking water.

\footnotetext{
"Towards a Healther Scotland" is available from The Stationery Office, price $£ 6$. ISBN 0-10-142692-5.
} 